\begin{tabular}{|l|l|l|l|l|}
\hline Cryst. Res. Technol. & $\mathbf{3 6}$ & 2001 & 3 & $295-301$ \\
\hline
\end{tabular}

\author{
N. M. GASANLY, A. AYDINLI*, Ö. SALIHOGLU \\ Department of Physics, Middle East Technical Universty, Ankara, Turkey \\ *Department of Physics, Bilkent University, Ankara, Turkey
}

\title{
Thermally Stimulated Current Observation of Trapping Centers in Undoped GaSe Layered Single Crystals
}

Undoped $p$-GaSe layered single crystals were grown using Bridgman technique. Thermally stimulated current measurements in the temperature range of $10-300 \mathrm{~K}$ were performed at a heating rate of 0.18 $\mathrm{K} / \mathrm{s}$. The analysis of the data revealed three trap levels at $0.02,0.10$ and $0.26 \mathrm{eV}$. The calculation for these traps yielded $8.8 \times 10^{-27}, 1.9 \times 10^{-25}$, and $3.2 \times 10^{-21} \mathrm{~cm}^{2}$ for capture cross sections and $3.2 \times 10^{14}$, $1.1 \times 10^{16}$, and $1.2 \times 10^{16} \mathrm{~cm}^{-3}$ for the concentrations, respectively.

Keywords: semiconductor compounds; layered crystals; GaSe; impurity levels; thermally stimulated current.

(Received October 26, 2000; Accepted February 26, 2001)

\section{Introduction}

GaSe is a member of the III-VI layered semiconductor family. It is a material of much importance in fields of both fundamental research and technical applications because of its structural, optical, electronic and photoelectronic properties. Investigations on the optical and electrical properties of this highly anisotropic compound revealed that it is a promising semiconductor for applications in photoelectronic devices in the visible region (KYAZYMZADE et al.). One of the determining factors in the eventual device performance of semiconductors is the presence of impurity and defect centers in the crystal. Thus, it is very useful to get detailed information on energetic and kinetic parameters of impurity centers in this semiconductor in order to obtain high quality devices.

Some published data on impurity levels in undoped GaSe crystals are available in the literature. Trap levels have been examined in nonintentionally doped $p$-type GaSe single crystals using photoinduced current transient spectroscopy measurements (MICOCCI et al. 1990). Two hole trapping levels at 0.20 and $0.80 \mathrm{eV}$ above the valence band have been observed. A systematic investigation of hole centers acting in $p$-GaSe single crystals has been carried out by means of thermally stimulated current (TSC) measurements in the range from 77 to $300 \mathrm{~K}$ (MANFREDOTTI et al. 1975). A series of hole centers has been found with energy depths ranging from 0.133 to $0.345 \mathrm{eV}$ above the valence band.

The purpose of the present work is to obtain further detailed information concerning deep and shallow traps in undoped GaSe crystals using the well-established technique of TSC measurements. In contrast with all previous TSC measurements on GaSe, we for the first time employ a wide temperature range of $10-300 \mathrm{~K}$. This temperature range allows us to check for the possibility of extremely shallow trap states. We report on the activation energy, the capture cross section, the type and concentration of the traps in GaSe. 


\section{Experimental}

Gallium selenide polycrystals were synthesized from high-purity elements (at least 99.999\%) taken in stoichiometric proportions. GaSe single crystals were grown by the modified Bridgman method. The analysis of X-ray diffraction data showed that they crystallize in hexagonal unit cell with parameters: $a=0.3745$ and $c=1.5921 \mathrm{~nm}$. The type of the GaSe crystals were found to be $p$-type after measuring with a hot probe. Crystals suitable for measurements were obtained by easy cleavage perpendicular to optical $c$-axis with following dimensions $5.0 \times 2.5 \times 0.3 \mathrm{~mm}^{3}$. The energy band gap of GaSe for indirect optical transition is $2.07 \mathrm{eV}$ at $10 \mathrm{~K}$ (AULICH et al.).

The electrodes were deposited by evaporating gold under vacuum, on both crystal surfaces according to sandwich geometry. Their thickness was about $100 \mathrm{~nm}$ on back side and $10 \mathrm{~nm}$ on front side, the latter corresponding to higher transmittance of the incident light. The sample was mounted in the cryostat with conducting silver paste. The backside was grounded through the sample holder. A thin gold wire was attached to the front side electrode by small droplet of silver paste. The I-V characteristics were checked to be ohmic and symmetric with respect to the polarity. Bias voltage of the order of $1 \mathrm{~V}$ was applied to the sample.

All measurements were carried out in vacuum in a „CTI-Cryogenics M-22,, closed-cycle helium system and were extended from 10 to $300 \mathrm{~K}$ with constant heating rate $\beta=0.18 \mathrm{~K} / \mathrm{s}$. The traps were filled by creating carriers with band-to-band photoexcitation of the samples. The light source was a $457.9 \mathrm{~nm}$ line $(2.715 \mathrm{eV}, 50 \mathrm{~mW})$ of „Spectra Physics,, argon laser. The thermally stimulated currents were measured by a "Keithley 619 " electrometer. The TSC and temperature data were stored in a personal computer.

In a typical experiment, the samples are cooled down to $\mathrm{T}=10 \mathrm{~K}$ and kept at this temperature for $\sim 10 \mathrm{~min}$. Then they are illuminated through the semi-transparent front electrode for a fixed period of time $(t=90 \mathrm{~s})$ at particular biasing condition and left to sit for $\sim 10 \mathrm{~min}$ to allow the photoconductivity signal to decay after exposure to light, and samples are then heated with a constant rate from 10 up to $300 \mathrm{~K}$.

\section{Results and discussion}

\subsection{Determination of the type of carrier traps}

Figure 1 shows the TSC spectra of GaSe single crystals for two biasing polarities and constant heating rate of $\beta=0.18 \mathrm{~K} / \mathrm{s}$ in the $10-300 \mathrm{~K}$ temperature range. When the sample is illuminated through a semitransparent contact, both types of carriers are created near the contact. Then depending on the bias voltage, only one type of carrier will be swept through the whole field zone, whereas the second type is collected very quickly. Therefore only the former can be trapped (MICOCCI et al. 1983). As is seen from Fig. 1, high-intensity overlapping peaks at a temperature of about $187 \mathrm{~K}$ appears only if the illumination occurs through the negatively biased contact. A low-intensity peak at about $85 \mathrm{~K}$ appears in both biasing polarities with small difference in intensity. Therefore, high-temperature peaks can be attributed to electron traps. As for the low-temperature current peak, it cannot be assigned simply as electron or hole trap.

\subsection{Activation energy and cross section determination}

In order to apply usual analytical methods for determination of crystal parameters it has been necessary to isolate TSC peaks. We used curve fitting method (COWELL, WOODS) for TSC 
spectra of GaSe crystal which revealed that only with three peaks, designated $T_{1}, T_{2}$, and $T_{3}$, do we get successfully fitting (Fig. 2).

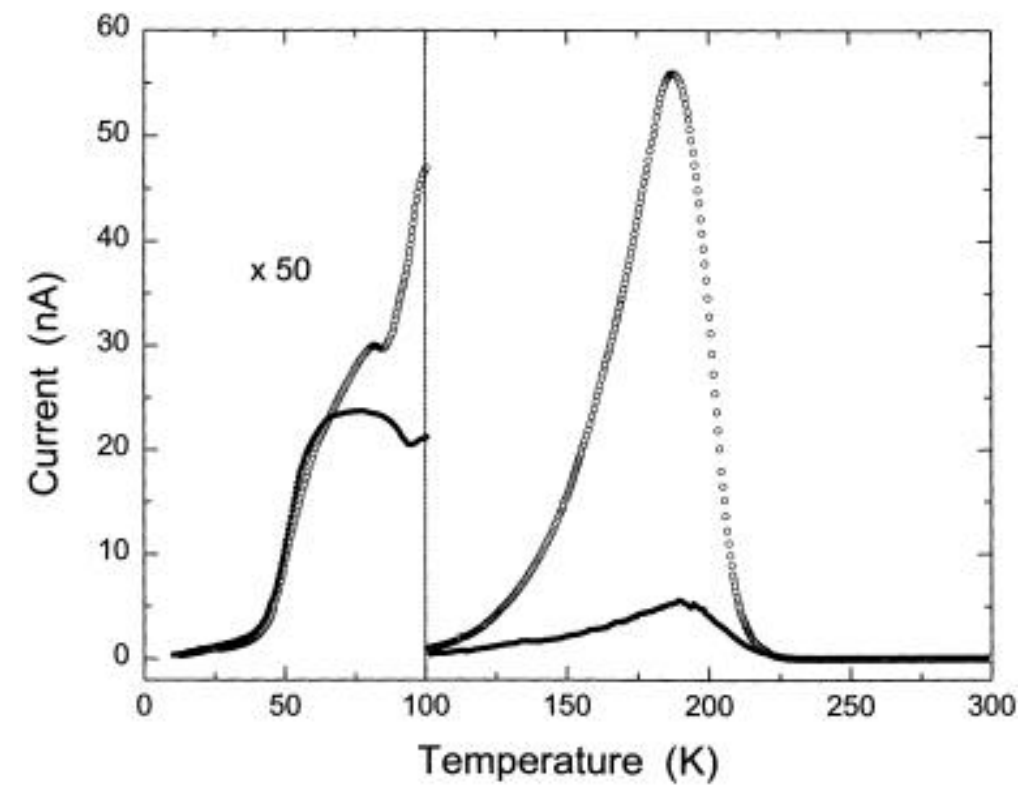

Fig. 1: Typical TSC curves of GaSe single crystal obtained under opposite bias voltage of $1 \mathrm{~V}$. Open and solid circles represent the experimental data obtained at illumination of negative and positive contacts, respectively.

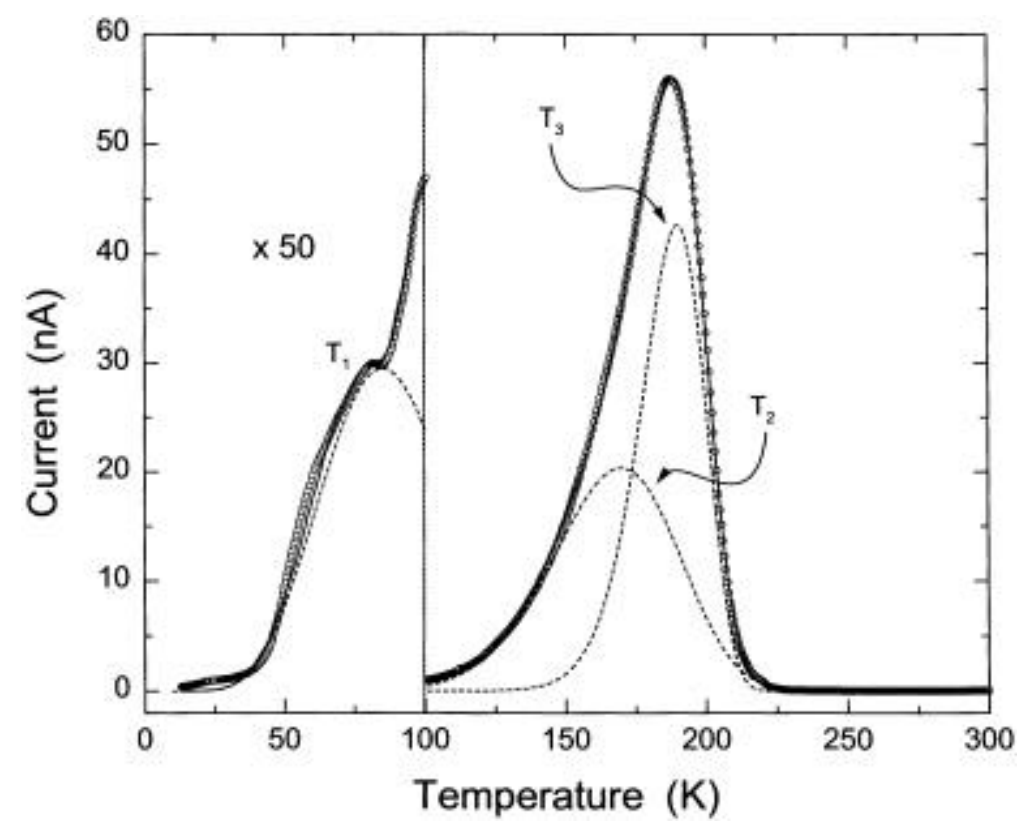

Fig. 2: Decomposition of the TSC spectrum of GaSe crystal into three separate peaks. Open circles are experimental data. Dotted curves represent decomposed peaks. Solid curve represents total fit to the experimental data. 
The overlapping TSC peaks were also isolated by applying the "thermal cleaning" procedure (CHEN, KIRSH) in order to verify the presence of $T_{2}$ and $T_{3}$. Thermal cleaning procedure was applied as follows: The sample was cooled and irradiated at $10 \mathrm{~K}$. The crystal was taken through the same heating cycle as before but was stopped at a temperature between $T_{2}$ and $T_{3}$. In this way, the traps responsible for the current peaks for $\mathrm{T}<\mathrm{T}_{3}$ were substantially emptied. The crystal was then recooled and reheated in the dark at the same constant rate. This allowed the observation of a sharper TSC peak at a slightly higher temperature due to electrons released from traps which are associated with the TSC maximum centered near $T_{3}$ (Fig. 3).

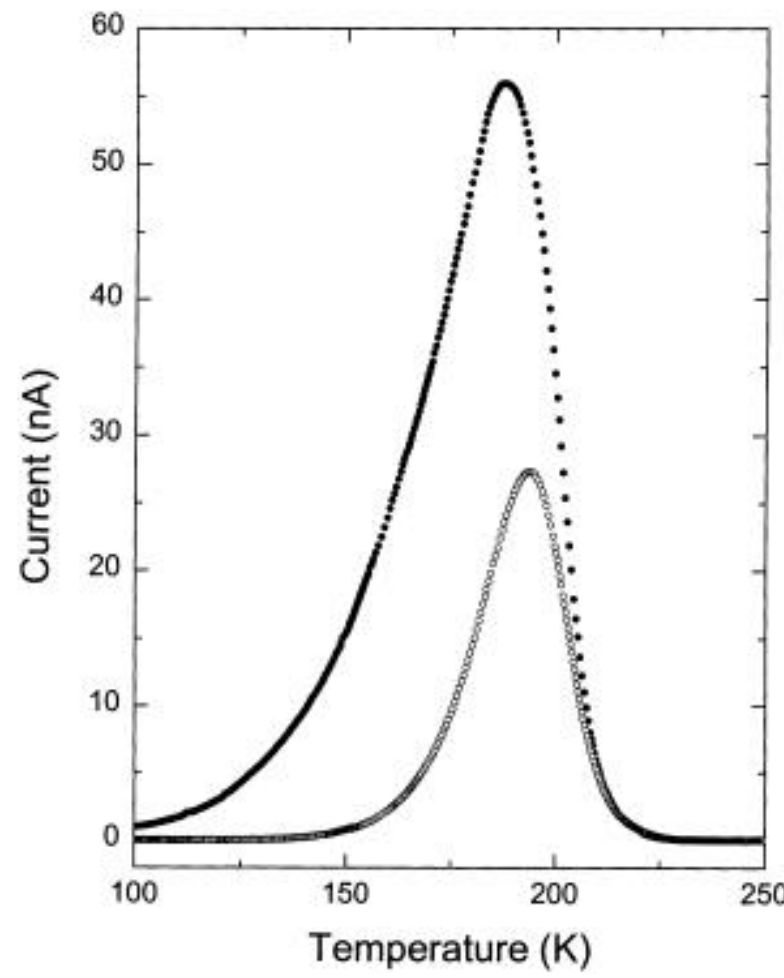

Fig. 3: The measured overlapping peaks $(T$ $\left.+T_{z}\right)$ (solid circles) and "thermally cleaned" peak $T_{3}$ (open circles).

There are several methods in literature to determine activation energy of trap from experimental TSC curves. We chose to use the „initial rise“, Chen's and curve fitting methods.

\subsection{1. "Initial rise" method}

The "initial rise" method originated by Garlick and Gibson (NICHOLAS, WOODS), and valid for all types of recombination kinetics, is based on the assumption that, when traps begin to empty as the temperature is increased, the TSC is proportional to $\exp \left(-E_{t} / k T\right)$. Here, $E_{t}$ is activation energy of the trap and $k$ is Boltzmann's constant. Thus a plot of the logarithm of the current flow against $1 / k T$ yields a straight line with a slope of $-E_{t}$, as shown in Fig 4 . The activation energies of the traps calculated by this procedure are found to be $0.02,0.09$, and $0.26 \mathrm{eV}$ for $T_{1}, T_{2}$, and $T_{3}$ peaks, respectively (Table 1 ). 
Fig. 4: Thermally stimulated current vs. $1 / k T$ for all three peaks in TSC spectrum of GaSe crystal. Solid lines represent the fits using "initial rise" method.

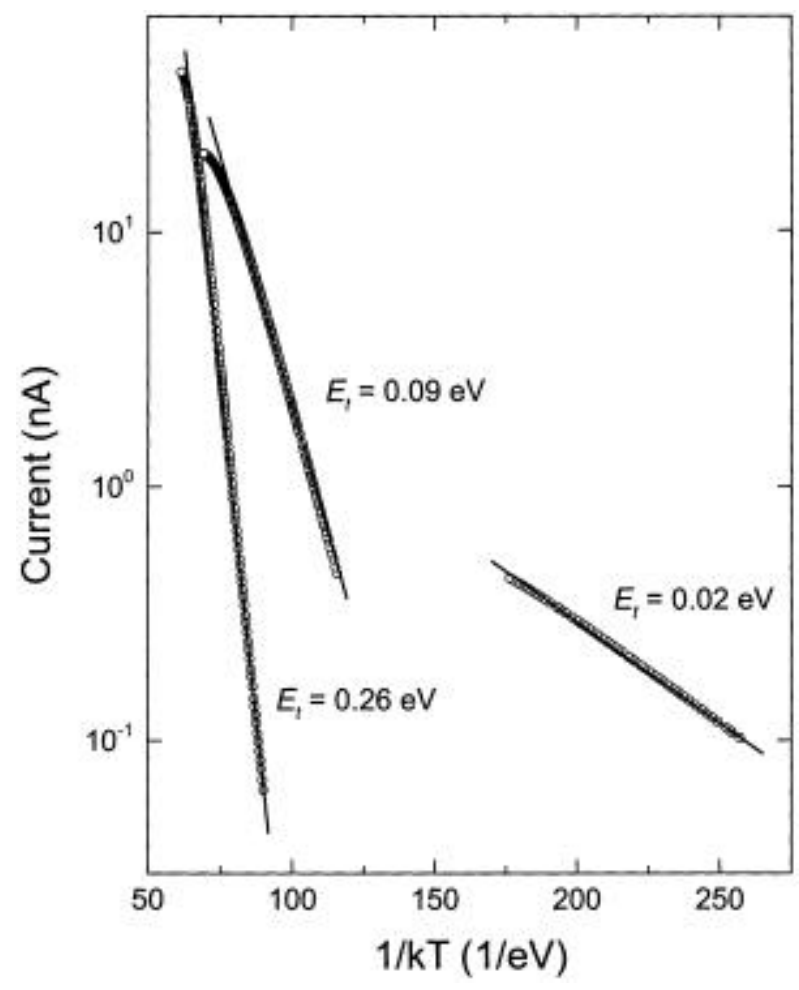

Table 1: The activation energy, capture cross section and concentration of traps for three TSC peaks of GaSe crystal.

\begin{tabular}{ccccccc}
\hline \multicolumn{7}{c}{$\mathrm{E}_{\mathrm{t}}(\mathrm{eV})$} \\
\hline Peak & $\mathrm{T}_{\mathrm{m}}(\mathrm{K})$ & Chen $\left(\mathrm{E}_{\tau}, \mathrm{E}_{\delta} \mathrm{E}_{\mathrm{w}}\right)$ & "Initial rise" & Curve fit & $\mathrm{S}_{\mathrm{t}}\left(\mathrm{cm}^{2}\right)$ & $\mathrm{N}_{\mathrm{t}}\left(\mathrm{cm}^{-3}\right)$ \\
\hline $\mathrm{T}_{1}$ & 85.7 & $0.01 ; 0.03 ; 0.02$ & 0.02 & 0.02 & $8.8 \times 10^{-27}$ & $3.2 \times 10^{14}$ \\
$\mathrm{~T}_{2}$ & 169.8 & $0.09 ; 0.13 ; 0.10$ & 0.09 & 0.10 & $1.9 \times 10^{-25}$ & $1.1 \times 10^{16}$ \\
$\mathrm{~T}_{3}$ & 190.0 & $0.25 ; 0.27 ; 0.26$ & 0.26 & 0.26 & $3.2 \times 10^{-21}$ & $1.2 \times 10^{16}$ \\
\hline
\end{tabular}

\subsubsection{Chen's method}

Chen's method requires the measurement of the low $T_{l}$ and high $T_{h}$ temperatures at which the TSC signal is equal to half of its maximum value. The activation energy of the trap is then given by CHEN

$$
\begin{aligned}
& E_{\tau}=\left[1.51+3.0\left(\mu_{g}-0.42\right)\right] k T^{2} m / \tau-\left[1.58+4.2\left(\mu_{g}-0.42\right)\right] 2 k T_{m}, \\
& E_{\delta}=\left[0.976+7.3\left(\mu_{g}-0.42\right)\right] k T^{2} m / \delta, \\
& E_{w}=\left[2.52+10.2\left(\mu_{g}-0.42\right)\right] k T^{2} m / w-2 k T_{m},
\end{aligned}
$$

where $\tau=T_{m}-T_{l}, \delta=T_{h}-T_{m}, w=\tau+\delta, \mu_{g}=\delta / w$, and $T_{m}$ is the temperature corresponding to TSC peak maximum. 
A value of $\mu_{g}=0.42$ was predicted by Chen for first order kinetics. Calculated values of $\mu_{g}$ for our decomposed peaks $T_{1}, T_{2}$, and $T_{3}$ were found to be $0.46,0.46$, and 0.42 , respectively. The activation energies obtained by this method are also reported in Table 1.

\subsubsection{Curve fitting method}

Under monomolecular conditions (i.e., no retrapping ) the TSC curve of a discrete set of traps with a trapping level $E_{t}$ below the conduction band is described by the equation (COWELL, WOODS)

$$
\sigma=n_{t} \tau e \mu \nu \exp \left[-E_{t} / k T-\int_{T_{0}}^{T}(v / \beta) \exp \left(-E_{t} / k T\right) d T\right),
$$

$\sigma$ is the thermally stimulated conductivity, $n_{t}$ the initial density of filled traps,$\tau$ the lifetime of a free electron, $\mu$ the electron mobility, $\beta$ the heating rate and $T_{o}$ the temperature from which heating begins following the filling of the traps. $v$ is the attempt-to-escape frequency of a trapped electron:

$$
\mathrm{V}=N_{c} \mathrm{v}_{t h} S_{t} \text {, }
$$

where $N_{c}=2\left(2 \pi m_{e}{ }_{e} k T / h^{2}\right)^{3 / 2}$ is the effective density of states in the conduction band , $v_{t h}$ the thermal velocity of a free electron and $S_{t}$ the capture cross section of the trap. If it is assumed that $v$ is independent on $T$ and that over the temperature span of the TSC curve, the variation of $\mu$ and $\tau$ with $T$ can be ignored, Eq. (1) can be rewritten approximately as:

$$
\sigma=A \exp \left[-t-B \exp (-t) t^{-2}\right]
$$

where $t=E_{t} / k T$, and $A$ and $B$ are constants : $A=n_{t} \tau e \mu \nu$ and $B=\nu E_{t} / \beta k$.

If Eq. (2) is differentiated and equated to zero to find the maximum of the curve, which occurs when $t=t_{m}=E_{t} / k T_{\mathrm{m}}$, then

$$
B=\exp \left(t_{m}\right) t_{m}^{3} /\left(t_{m}+2\right) .
$$

After finding $t_{m}$, we can obtain $v$, using $B=v E_{t} / \beta k$, and then calculate $S_{t}$ from $v=N_{c} v_{t h} S_{t}$.

In TSC spectra of GaSe we observed three peaks, $T_{1}, T_{2}$, and $T_{3}$, with $T_{2}$ and $T_{3}$ overlapping each other. Therefore the following fit function was used:

$$
\sigma_{\text {total }}=\sum \sigma_{\mathrm{m}}
$$

This allowed us to obtain $\mathrm{E}_{\mathrm{t}}$ for each peak directly from the fit. Then we calculated $S_{t}$ for all three peaks (Table 1).

\subsection{Trap concentration determiation}

The concentration of the traps was estimated using the relation (MANFREDOTTI et al. 1976)

$$
N_{T}=Q / A L \text { e } G
$$

Here $Q$ is the quantity of charge released during a TSC experiment and can be calculated from the area under the TSC peaks; $A$ and $L$ are the area and the thickness of the sample , respectively; $e$ is the electronic charge and $G$ is the photoconductivity gain, which equals to 
the number of electrons passing through the sample for each absorbed photon. $N_{T}$ was calculated by assuming $G=1$ (MANFREDOTTI et al. 1976). The obtained values of $N_{T}$ are presented in Table 1.

\section{Conclusions}

TSC spectroscopy has been used to characterize traps in undoped GaSe layered crystals. Three traps were observed in the temperature range from 10 to $300 \mathrm{~K}$ using excitation light of $2.715 \mathrm{eV}$, a sufficiently long illumination time $(\sim 10 \mathrm{~min})$ and an heating rate of $0.18 \mathrm{~K} / \mathrm{s}$. Since, current polarity and intensity alters by changing the bias polarity, $T_{2}$ and $T_{3}$ peaks are assigned to electron traps. $T_{1}$ peak might be related to either hole or electron trapping. The energy levels for $T_{1}, T_{2}$ and $T_{3}$ peaks are $0.02,0.10$ and $0.26 \mathrm{eV}$, respectively, as determined by curve fitting method. The activation energies of peaks, evaluated also using ,initial rise“ and Chen's methods, are in agreement with each other, within the accuracy of the methods used. We note that this is the first time a shallow trap level of $0.02 \mathrm{eV}$ has been observed in GaSe due mainly to low initial temperatures used in this work. The capture cross section of the traps are calculated to be $8.8 \times 10^{-27}, 1.9 \times 10^{-25}$, and $3.2 \times 10^{-21} \mathrm{~cm}^{2}$. Also the concentration of the traps are estimated to be $3.2 \times 10^{14}, 1.1 \times 10^{16}$, and $1.2 \times 10^{16} \mathrm{~cm}^{-3}$.

\section{References}

Aulich, E., Brebner, J., Mooser, E.: Phys. Stat. Sol. 31 (1969) 129

CHEN, R.: J. Appl. Phys. 40 (1969) 570

Chen, R., KIRSH, Y.: Analysis of Thermally Stimulated Processes, Pergamon Press, Oxford (1981), p. 275.

Cowell, T. A. T., Woods, J.: Brith. J. Appl. Phys. 18 (1967) 1045

Kyazymzade, A., Mekhtieva, N., Akhmedov, A.: Sov. Phys. Semicond. 25 (1992) 90

Manfredotti, C., Murri, R., Quirini, A., Vasanelli, L.: Phys. Stat. Sol. 38 (1976) 685

Manfredotti, C., Rizzo, A., Blasi, C., Gallassini, S., Ruggiero, L.: J. Appl. Phys. 46 (1975) 4531

Micocci, G., Rizzo, A., Tepore, A.: J. Appl. Phys. 54 (1983) 924

Micocci, G., Siciliano, P., Tepore, A.: J. Appl. Phys. 67 (1990) 6581

Nicholas, K. H., Woods, J.: Brith. J. Appl. Phys. 15 (1964) 783

\section{Contact information:}

Prof. Dr. N. M. GASANLY*

Ö. SALIHOGLU

Department of Physics

Middle East Technical Universty

06531 Ankara

Turkey

Prof. Dr. A. AydinLI

Department of Physics

Bilkent University

06533 Ankara

Turkey

*corresponding author

on leave from Physics Department, Baku State University, Baku, Azerbaijan

e-mail: nizami@metu.edu.tr 